\title{
Adaptive multiresolution analysis based on synchronization
}

\author{
Meyer Z. Pesenson \\ Computing and Mathematical Sciences Department, Caltech, Pasadena, California 91125, USA \\ Isaac Z. Pesenson \\ Department of Mathematics, Temple University, Philadelphia, Pennsylvania 19122, USA
}

(Received 14 June 2011; published 11 October 2011)

\begin{abstract}
We propose an adaptive multiscale approach to data analysis based on synchronization. The approach is nonlinear, data driven in the sense that it does not rely on a priori chosen basis, and automatically determines the data scale. Numerical results for one- and two-dimensional cases illustrate that the method works effectively for the usual modulated signals such as chirps, etc., as well as for more complicated data with multiple scales. The method extends straightforwardly to functions defined on weighted graphs and grids in high dimensions. Connections with some other recent approaches to multiscale analysis are briefly discussed.
\end{abstract}

DOI: 10.1103/PhysRevE.84.045202

PACS number(s): 05.45.Xt, 07.05.Kf, 02.30.Mv, 07.05.Rm

Modern scientific instruments generate new data types, such as data defined on graphs and manifolds, and vector and tensor data [1]. These complex data sets cannot be adequately understood without detecting various scales that might be present in the data. Thus, the development of multiscale analysis methods applicable to these more general settings is of great importance $[1,2]$. The wavelet transform is particularly suited to the analysis of different scales in data. Though each type of wavelet provides its own effective analysis, the need to choose a basis a priori makes the method not fully adaptive [3-5]. The so-called Hilbert-Huang transform (HHT), which combines the empirical mode decomposition (EMD) with the Hilbert transform (HT), was introduced to avoid dealing with a predetermined basis and to calculate the instantaneous frequency and amplitude (see Ref. [3] and references there). Recent works [4,5] suggest important modifications of EMD and their mathematical justifications. The so-called synchrosqueezed wavelet transform was developed in work [5]. These methods are restricted mostly to one-dimensional (1D) or two-dimensional (2D) signals. At the same time, besides the importance of the modern data defined on graphs, the graph representation of structured data provides a fruitful model for the relational data mining process [1]. Moreover, a weighted graph also provides a discrete "representation" of a Riemannian manifold, e.g., the weighted Laplacian can be considered as a discrete analog of the Laplace-Beltrami operator, and analysis of graph functions has important applications to image processing, machine learning, discretization schemes, meshes, sampling and approximation theory, etc. [1,2].

In order to develop a multiscale analysis of functions defined on graphs we propose to use the concept of synchronization. In fact, approaches to data analysis problems are sometimes motivated by physics, and synchronization in particular has been used for data clustering [6]. The approach is usually based on the so-called phase model. Near the regime, where the individual phases are close to each other, this formulation reduces to the spectral analysis of the combinatorial Laplacian, thus being closely related to well-known approaches such as normalized cuts, eigenmaps, and diffusion maps [7]. Our approach to a multiscale analysis of a function defined on a weighted graph starts by associating with the nodes of the graph a set of coupled limit-cycle oscillators. Let us consider a weighted graph $G=(V, W)$ with a set $V$ of $N$ vertices, together with a set $W$ of weights, and a vector-valued function $\Omega^{0}: V \rightarrow \mathbf{R}^{m}$; here, for simplicity, we will be concerned only with $m=1$, though a more general $m>1$ can also be examined. One can introduce the combinatorial Laplacian $L$, with $L_{k, j}=A_{k j}-d_{k} \delta_{k j}$, where $A$ is a symmetric adjacency matrix $N \times N$ whose components are the weights, and the diagonal degree matrix has the elements $d_{k}=\sum_{j=1}^{N} A_{k j}$. In order to analyze the function $\Omega^{0}\left(V_{k}\right)$, we study synchronization in the following system of the space-discrete Ginzburg-Landau (GL) equations coupled diffusively

$$
d U_{k} / d t-\left(1+i \omega_{k}\right) U_{k}+\left|U_{k}\right|^{2} U_{k}-c \sum_{j}^{N} L_{k j} U_{j}=0,
$$

where $U_{k}(t), k=1, \ldots, N$, describes the state of the $k$ th oscillator at time $t, \omega_{k}$ are the intrinsic frequencies of the individual oscillators, and parameter $c$ is the coupling strength. We are assuming that the distribution of the intrinsic frequencies is given by the function $\Omega^{0}: \omega_{k}=\Omega^{0}\left(V_{k}\right)$. One rationale for this approach is that for a given coupling strength, oscillators with close intrinsic frequencies synchronize first, thus capturing (for a "good" sampling) the curvature of the function $\Omega^{0}\left(V_{k}\right)$. Therefore, both the topology of the graph (the weights) and the geometry of the function are taken into account in such a description.

For a simple distribution of the intrinsic frequencies in one dimension, one can do a quick estimate of the asymptotic solution, as $t \rightarrow \infty$, by using the phase dynamics and considering the continuous limit. In this approximation the evolution of the phase $\psi(x, t)$ can be described by a Burgers-type equation (see Ref. [6]; a general equation for the phase evolution of strongly nonlinear waves in high dimensions was derived in Ref. [8]) $\partial \psi / \partial t-a(\partial \psi / \partial x)^{2}-D \partial^{2} \psi / \partial x^{2}=\Omega^{0}(x)$, where $\Omega^{0}(x)$ is a one-dimensional frequency distribution. The Cole-Hopf transform reduces this equation to an inhomogeneous heat equation. It can be found from the usual eigenvalue problem with the potential proportional to $\Omega^{0}(x)$ that, for $\Omega^{0}(x)$ with a local maximum $\Omega_{\max }^{0}$ and $\Omega^{0}(x) \rightarrow \Omega_{\lim }^{0}$ as $x \rightarrow \pm \infty$, an asymptotic solution is the one with a frequency $\Omega_{\text {select }}^{0}$, which satisfies $\Omega_{\text {lim }}^{0}<\Omega_{\text {select }}^{0}<\Omega_{\max }^{0}$. 


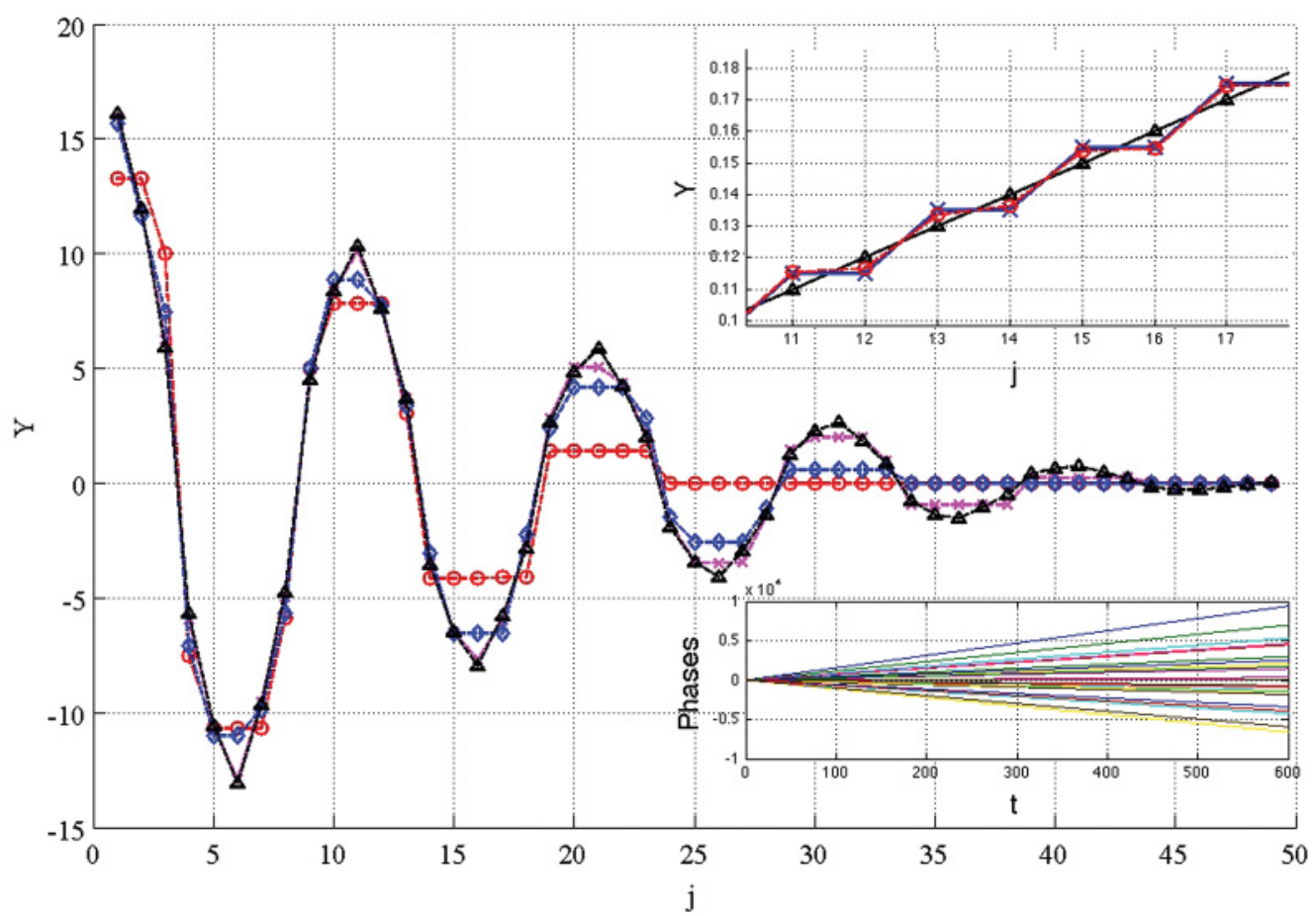

FIG. 1. (Color online) Synchronization and Haar wavelets [axes: for the 1D case the oscillators are ordered in space and $j$ is the spatial coordinate of oscillators; $\left.Y=\Omega^{0}\left(V_{k}\right)\right]$. Various stages $n$ of synchronization-based MRA: $Y=\bar{\Omega}^{10}\left(V_{k}\right)(\times$ symbols $) ; Y=\bar{\Omega}^{100}\left(V_{k}\right)$ (diamonds); $Y=\bar{\Omega}^{500}\left(V_{k}\right)$ (circles); $c=0.25$. Top inset: Weighted averaging of the individual frequencies for a linear distribution of intrinsic frequencies - the signal (triangles), the Haar averaged signal $A 2$ ( $\times$ symbols), synchronized frequencies (circles). Bottom inset: The phases uniformly grow in time (axes: phases vs time).

In order to study synchronization phenomena in general oscillatory systems, the phase has to be defined first. Defining the phase may not be a straightforward task, but the system of oscillators (1) has attractors with simple topology (the so-called phase coherent attractors), so by introducing $U_{k}(t)=$ $u_{k}(t) \exp \left[i \varphi_{k}(t)\right]$ one can define $\varphi_{k}(t)$ as the phases, and determine the mean frequencies

$$
\bar{\Omega}_{k}=\left\langle d \varphi_{k} / d t\right\rangle=\lim _{t \rightarrow \infty}\left\{\left[\varphi_{k}\left(t+t_{0}\right)-\varphi_{k}\left(t_{0}\right)\right] / t\right\} .
$$

We started by solving the system (1) for various functions $\Omega^{0}\left(V_{k}\right)$, with homogeneous initial conditions plus small random perturbations, and calculating the mean frequencies [Figs. 1, 2(a), and 3]. To make sure that for a given coupling strength the integration time was sufficient for reaching the asymptotic solutions $(t \rightarrow \infty)$, we varied the integration time and found that an increase of it by a factor of $10^{2}$ resulted only in differences of less than $1 \%$. Also, to illustrate that the phases $\varphi_{k}(t)$ are approximately monotonous functions of time, we plotted them in Fig. 1 (bottom inset). For these phases the error of the numerical differentiation in (2) is less than $0.1 \%$, so formula (2) provides a robust definition of the frequency of oscillators. Synchronization is detected by finding the mean frequencies that satisfy the inequality $\left|\bar{\Omega}_{k}-\bar{\Omega}_{l}\right| \leqslant \epsilon$ (where $\epsilon$ is a small threshold), and it may visually be identified as plateaus in the oscillator's mean frequency versus oscillator's space location plots [Figs. 1-5]. As anticipated, synchronization occurs first near the local extrema [Figs. 1 (main panel), 2(a), and 4]. Figure 2 demonstrates the application of this approach to a chirp signal, while Fig. 3 shows a comparison of two different chirps and a harmonic signal. One can see from Fig. 3(b) that the lengths and pattern of the plateaus capture the differences between the signals: the length and the height of the plateaus of the harmonic signal $(x$ symbols $)$ are constant, while the scales and the heights corresponding to the chirps track the frequency variations of the chirps. One can also see from Figs. 4 and 5 that the scale and the distribution (or pattern) of the synchronization plateaus for 1D and 2D multiscale signals adequately represent the structure of such signals.

To perform a multiscale analysis of the function $\Omega^{0}\left(V_{k}\right)$, we solve the system (1) in a progression of steps, or stages, starting with $\Omega^{0}\left(V_{k}\right)$ as the frequency distribution for the first stage. For each following stage $n$ we solve the system (1) with a frequency distribution $\bar{\Omega}^{n-1}\left(V_{k}\right)$ obtained by applying formula (2) to the solution from the previous stage. The calculation continues until the difference between distributions of the mean frequencies obtained from the solutions of two consecutive steps is less than a certain threshold. At every stage, the length of each synchronization plateau gives the local scale of the function. Each stage gives a more coarse local scale of the data than the one obtained at the previous stage, or in other words, each stage generates a coarser rendering of the function $\Omega^{0}\left(V_{k}\right)$ [Figs. 1 (main panel) and 4]. The distribution of frequencies $\bar{\Omega}^{n}\left(V_{k}\right)$ forms the $n$th level of the multiresolution analysis (MRA) of the function $\Omega^{0}\left(V_{k}\right)$. Unlike the linear approaches to MRA, this method does not 

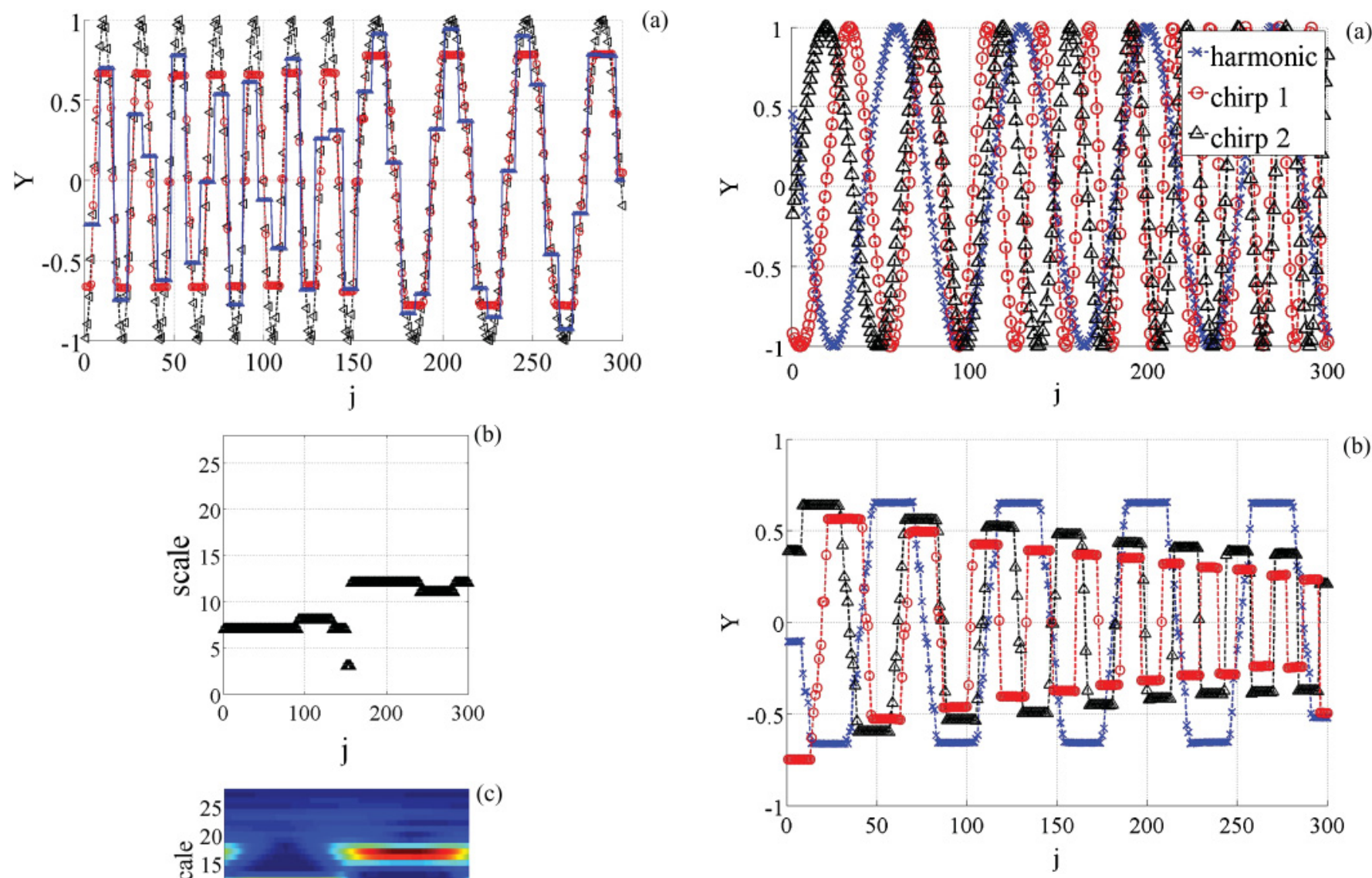

(b)

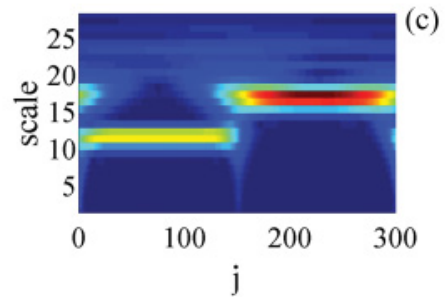

FIG. 3. (Color online) Two chirps and a harmonic signal [axes: $j$ is the spatial coordinate; $Y=\Omega^{0}\left(V_{k}\right)$ ]. (a) Chirp No. 1 [triangles (up)]; chirp No. 2 (circles); harmonic signal ( $\times$ symbols). (b) The corresponding synchronized frequencies. Note that the length and the height of the plateaus corresponding to the harmonic signal are constant, while the scale and the height of the plateaus corresponding to the chirps [triangles (up), circles] reflect the frequency changes of the chirps.

[triangles (left)]; the averaged Haar signal $A 4$ [triangles (up)]; the synchronization plateaus (circles). (b) The synchronized scales (the edge effects are neglected). (c) The continuous Morlet wavelet transform of $\Omega^{0}\left(V_{k}\right)$. Synchronization gives a sharper overall value of the scale, comparing to the continuous Morlet wavelet transform.

rely on a preassigned family of templates generated from a basic one by dilations and translations (see the discussion in Ref. [5]), and instead gives a variable local scale for every stage.

A few remarks are in order. An optimal choice of the parameter $c$ will facilitate the computation process by reducing the number of stages, and should depend on the smallest scale of the data analyzed. Another point is that a change in the initial conditions leads to different solutions. As the numerical experiments show, this is analogous, for our purposes, to the issue of choosing a value of $c$ for the first stage. Indeed, a change in the initial conditions by a factor of $10^{3}$ does not affect the asymptotic averaged frequency distribution very much, and it can be shown that the same averaged frequencies could have also been obtained for the given initial condition with a slightly different value of the parameter $c$. The juxtaposition of the synchronized frequencies with the Haar wavelet averaged

signals in Fig. 1 (top inset), and Fig. 2(a) demonstrates a certain similarity between the synchronization averaging and MRA performed by discrete wavelet transforms [a comparison with the continuous Morlet wavelet transform in Fig. 2(b,c) shows that synchronization gives overall a sharper value of the scale]. Indeed, it is known that the phase-locked frequency is in general a nondecreasing function of a weighted average of individual frequencies, with the weights depending on the parameters of the system [9] [Figs. 1 (main panel), 2(a), and 4]. In other words, similarly to discrete wavelet transforms, the approach based on synchronization provides a multiscale hierarchical decomposition of the function $\Omega^{0}\left(V_{k}\right)$ into the averaged modes. These features suggest that the approach based on synchronization is related to wavelets and we call it adaptive synchronization wavelets.

There are several links between the synchronization-based approach and the recent approaches to MRA developed in Refs. [3-5]. For example, the fact that the amplitude of the data oscillations approaches the local mean on a certain scale as the number of stages increases corresponds to the behavior of the higher intrinsic modes of Refs. [3-5]. Also, the synchronized 


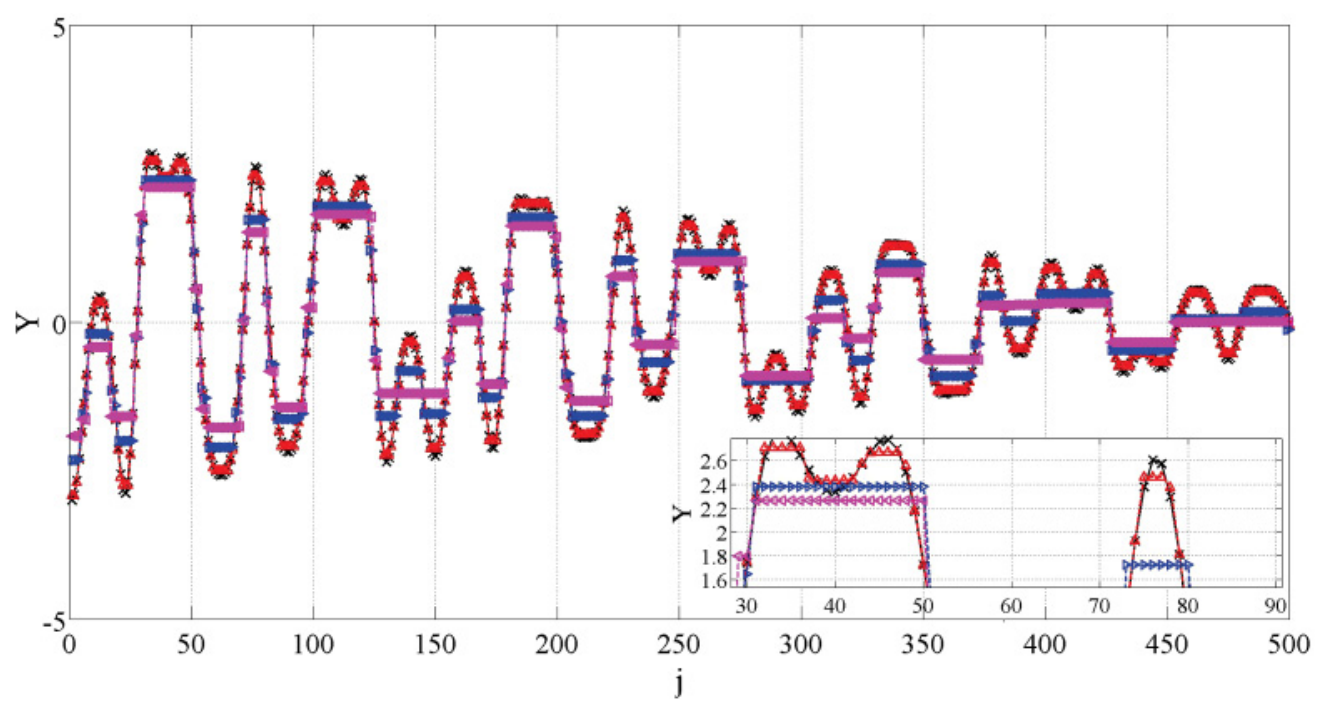

FIG. 4. (Color online) Multiscale analysis based on synchronization [axes: $j$ is the spatial coordinate; $\left.Y=\Omega^{0}\left(V_{k}\right)\right]$. Main figure: data with multiple scales (× symbols); various stages $n$ of synchronization-based MRA: $Y=\bar{\Omega}^{1}\left(V_{k}\right)$ [triangles (up)]; $Y=\bar{\Omega}^{40}\left(V_{k}\right)$ [triangles (right)], $Y=\bar{\Omega}^{80}\left(V_{k}\right)$ [triangles (left)]. Inset: A zoomed in version of the main figure.

frequencies trace the envelope of the function $\Omega^{0}\left(V_{k}\right)$ as a result of synchronization taking place first near the local extrema of $\Omega^{0}\left(V_{k}\right)$, and this is analogous to the HHT method which constructs the upper and lower envelopes based on the local extrema points [3]. HT is the keystone of HHT [3] and it provides another link between these two approaches. Indeed, the so-called analytic signal approach is also based on HT, and when it is applied to synchronization, it gives practically the same result (for "good" systems) as the mean frequency approach (2) [6,10]. At the same time, the synchronization approach and HHT are different in the sense that the latter one applies HT to the extrinsic modes extracted by EMD from the function $\Omega^{0}\left(V_{k}\right)$, while the former one determines the local scale from the solutions of the nonlinear differential equations (1) with $\Omega^{0}\left(V_{k}\right)$ being the "potential" at the first stage (the terminology comes from the 1D case considered above, where the Cole-Hopf transform led to the linear eigenvalue problem).

We have introduced a unique approach to multiresolution analysis based on synchronization. It is an adaptive, nonlinear method that does not rely on a priori chosen basis and automatically determines the data scale. It can be considered as a transform based on the nonlinear differential equations (1). The main advantage of this approach is that it can straightforwardly be extended to nonuniformly sampled data, high-dimensional settings, and, more generally, to data defined on graphs or point clouds with nonlocal weighted edges, where traditional methods are not available.
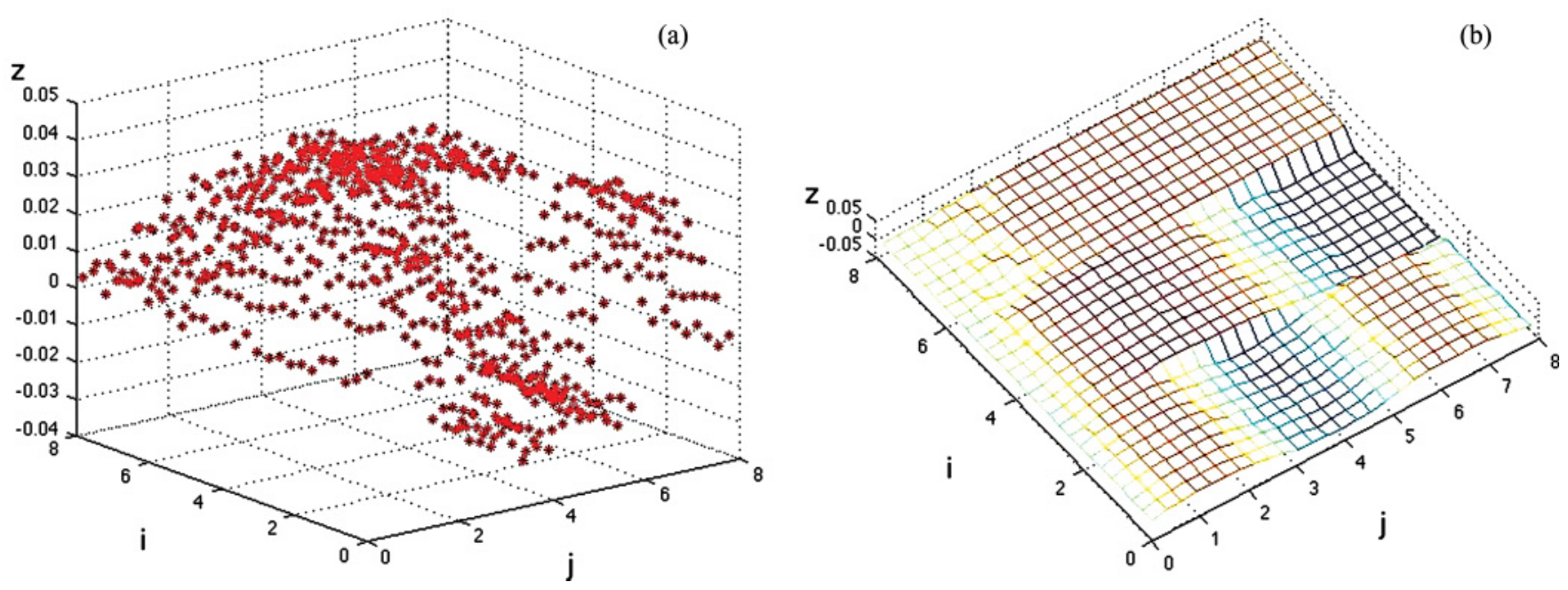

FIG. 5. (Color online) A function $\Omega^{0}\left(V_{k}\right)$ defined on a 2D grid (sampled from harmonics with three different wave numbers). (a) The data [axes: $i, j$ are the spatial coordinates, $z=\Omega^{0}\left(V_{k}\right)$ ]. (b) The scales in the data revealed by synchronization [axes: $i, j$ are the spatial coordinates, $z=\bar{\Omega}^{6}\left(V_{k}\right)$; colors are proportional to the function values, with darker colors corresponding to extrema. 
M.P. would like to thank M. Desbrun and J. Tropp for the stimulating discussions and suggestions that helped to improve the paper. We thank the anonymous reviewers for constructive suggestions that helped to im- prove the presentation. This work was in part supported by the grant from NGA, NURI HM1582-08-10019, and the grant from AFOSR, MURI FA9550-09-10643 .
[1] I. Rahman, I. Drori, V. Stodden, D. Donoho, and P. Schröder, SIAM Multiscale Model. Simul. 4, 1201 (2005); M. Gavish, B. Nadler, and R. Coifman, in Proceedings of the 27th International Conference on Machine Learning (ICML-10), edited by Johannes Fürnkranz and Thorsten Joachims (Omnipress, 2010), pp. 367-374; M. Pesenson, I. Pesenson, and B. McCollum, Adv. Astron. 350891 (2010).

[2] For wavelets and signal/image processing, see I. Daubechies, Ten Lectures on Wavelets (SIAM, Philadelphia, 1992); S. Mallat, A Wavelet Tour of Signal Processing: The Sparse Way, 3rd ed. (Academic, Boston, MA, 2009). For machine learning applications, see T. Hastie, R. Tibshirani, and J. Friedman, The Elements of Statistical Learning: Data Mining, Inference, and Prediction, 2nd ed. (Springer, Berlin, 2009). For geometric discretization and adaptive, irregular meshes, see D. Pavlov, P. Mullen, Y. Tong, E. Kanso, J. E. Marsden, and M. Desbrun, Physica D: Nonlinear Phenomena 240, 443 (2011). For convergence of the combinatorial Laplacian to the Laplace-Beltrami operator, see K. Fujiwara, Proc. Am. Math. Soc. 123, 2585 (1995); M. Hein, J.-Y. Audibert, and U. von Luxburg, J. Mach. Learn. Res. 8, 1325 (2007). For efficient sampling and sparse signals, see J. Tropp, J. Laska, M. Duarte, J. Romberg, and R. Baraniuk, IEEE Trans. Inf. Theory 56, 520 (2010). For localized frames on general compact homogeneous manifolds, see D. Geller and I. Pesenson, J. Geom. Anal. 21, 334 (2011).

[3] N. Huang et al., Proc. R. Soc. London A 454, 903 (1998); A. Ayenu-Prah, N. Attoh-Okine, and N. Huang, in Transforms and Applications, 3rd ed., edited by A. Poularikas (CRC, Boca Raton, FL, 2010).
[4] T. Hou, M. Yan, and Z. Wu, Adv. Adapt. Data Anal. 1, 483 (2009).

[5] I. Daubechies, J. Lu, and H.-T. Wu, Appl. Comput. Harmon. Anal. 30, 243 (2011).

[6] A. Pikovsky, M. Rosenblum, and J. Kurths, Synchronization. A Universal Concept in Nonlinear Science (Cambridge University Press, Cambridge, UK, 2001); S. Fortunato, Phys. Rep. 486, 75 (2010).

[7] For spectral clustering and diffusion on graphs, see J. Shi and J. Malik, IEEE Trans. Pattern Anal. Mach. Intell. 22, 888 (2000); R. Kondor and J. Lafferty, in Proceedings of the 2002 International Conference on Machine Learning and Applications-ICMLA (CSREA Press, Las Vegas, Nevada, USA, 2002), p. 315; M. Belkin and P. Niyogi, Neural Computation 15, 1373 (2003); R. Coifman and S. Lafon, Appl. Comput. Harmon. Anal. 21, 5 (2006). For reviews of common methods for dimensionality reduction and semisupervised learning for functions defined on graphs by using the graph Laplacian, see M.-A. Belabbas and P. Wolfe, Philos. Trans. R. Soc. A 367, 4295 (2009); O. Chapelle, B. Schölkopf, and A. Zien, Semi-Supervised Learning (MIT Press, Cambridge, MA, 2006).

[8] M. Pesenson, Phys. Fluids A 3, 3001 (1991); V. Shrira and M. Pesenson, in Nonlinear and Turbulent Processes in Physics, edited by R. Sagdeev, Vol. 2 (Harwood Academic, New York, 1983), p. 1061.

[9] I. Blekhman, Synchronization in Science and Technology (ASME, New York, 1988).

[10] H. Schuster and W. Just, Deterministic Chaos, 4th ed. (Wiley, Weinheim, 2005). 